\title{
GAIN AND NOISE FIGURE ANALYSIS OF ERBIUM DOPED FIBER
}

\author{
AMPLIFIERS \\ Swapandeep Kaur ${ }^{1}$, Prabhjot Singh Sandhu ${ }^{2}$ \\ ${ }^{1}$ M tech, Electronics and Communication, Chandigarh Engineering College, Punjab, India \\ ${ }^{2}$ Assistant Professor, Electronics and Communication, Chandigarh Engineering College, Punjab, India
}

\begin{abstract}
Erbium doped fiber amplifier (EDFA) performance is dependent on several factors such as fiber length, pump power, Er3+ concentration. This paper involves the simulation of an EDFA using Optisystem and analyzes the gain and noise figure of EDFA in the Conventional band in terms of pump power and fiber length. The gain increases initially with the pump power when the length is fixed and then it decreases. The gain also increases with the length when pump power is fixed and decreases after reaching a maximum. Whereas the noise figure increases with length and decreases with pump power.
\end{abstract}

Key Words: EDFA, pump power, gain, noise figure

\section{INTRODUCTION}

Optical amplifiers are devices which can amplify the optical signal directly without electrical to optical and optical to electrical conversion. Their operation is the same as that of a lasing device. The only difference is that it is not having any feedback of the signal as lasers use.

The two main kinds of optical amplifiers are semiconductor optical amplifier (SOA) and rare earth amplifiers. SOA are based on the principle of stimulated emission to amplify optical information signal while Gain in fiber amplifiers is either provided by stimulated Raman or Brillouin scattering or by rare earth dopants like erbium.

Optical amplifiers are used in general applications like inline amplifiers, preamplifiers and power or booster amplifiers and also in crucial applications to carry information over long distances. They are used in WDM networks.

EDFA is an amplifier that is best used because of its low loss and high gain. For communication, there are two windows 1530-1560nm(C-band) and 1560-1610nm (Lband).

\section{DOPED FIBER AMPLIFIERS}

Doped fiber amplifiers are devices which consist of a doped optical fiber as a gain medium for amplification of signal. The input and the pump signal are directed into the fiber and the amplification takes place when the doping ions interact. EDFA is a well known example. Here the doping of silica core is done with Er3+ .It could be pumped effectively at wavelengths of $980 \mathrm{~nm}$ or $1480 \mathrm{~nm}$ and displays gain in the $1550 \mathrm{~nm}$ region.

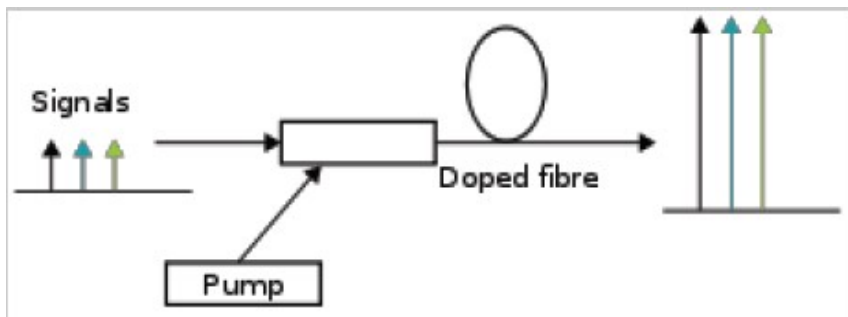

Fig 1 Symbolic diagram of a simple Doped fiber Amplifier

\section{BASIC PRINCIPLE OF EDFA}

The principle of operation is stimulated emission which involves mixing of a very high-power ray of light with the input signal using a wavelength selective coupler. The input signal and the pump light are defined by separate wavelengths. The mixture of light is then directed in the fiber whose core is doped with erbium ions $(\mathrm{Er} 3+)$.The Er3+ ions are excited to their higher energy state by the pump light. Since the higher levels are unstable, the electrons have a tendency to come back to the ground state .If the time taken by the electrons to come to the ground state from a particular level is long as compared to the lifetime of the previous state that led to the level, a metastable level is formed and electrons will accumulate here. If the number of electrons in the metastable band is more than in the ground state, population inversion will occur. This is essential for amplification to take place.

The input signal and the pump signal are at a separate wavelength and when the photons of the signal come into presence of the excited erbium ions, they release some of the energy to the signal and return to the lower-energy state. The energy is given in the form of supplementary photons that have the identical phase and direction as the original signal that is being amplified. Hence they are synchronous and coherent to each other. An isolator is usually put at the output so as to stop reflections returning from the fastened 
fiber because reflections can interrupt the operation of amplifier and in the utmost instance could lead to the amplifier to act as a laser.

Edfa can operate both in forward pumping as well as backward pumping configuration.In forward pumping,signal and pump co-propagate with each other and in backward configuration they counter- propagate with each other.Forward pumping produces less noise and less output power whereas backward pumping is noisier producing higher output power.A compromise can be made by combining both these geometries in a bi-directional configuration.

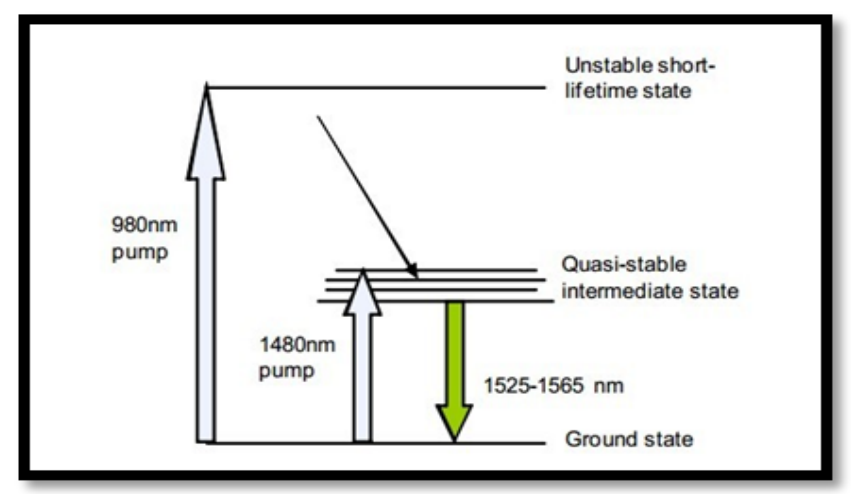

Fig 2 Energy levels of the erbium

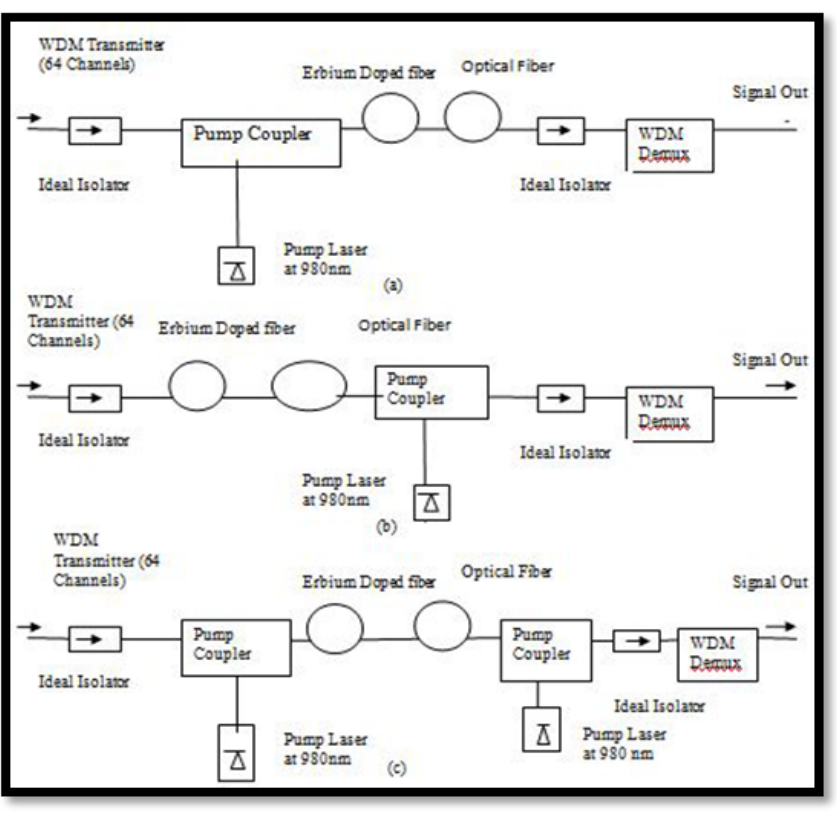

Fig 3 Block Diagram of three pumping techniques (a) CoPumping (b) Counter- Pumping (c) Bidirectional Pumping

\section{SIMULATION}

An EDFA model proposed by Giles is used for the analysis. The circuit diagram is shown in figure 4.In this paper, we are studying the analysis of gain and noise figure. The pump power is set at $980 \mathrm{~nm}$. The EDFA parameters are fixed which are shown in figure 5. The input signal wavelength is set at $1550 \mathrm{~nm}$ and input power is set at $-40 \mathrm{dbm}$.Optical Spectrum Analyzer, Optical Power Meter and Dual Port Power meter are used as the measurement components

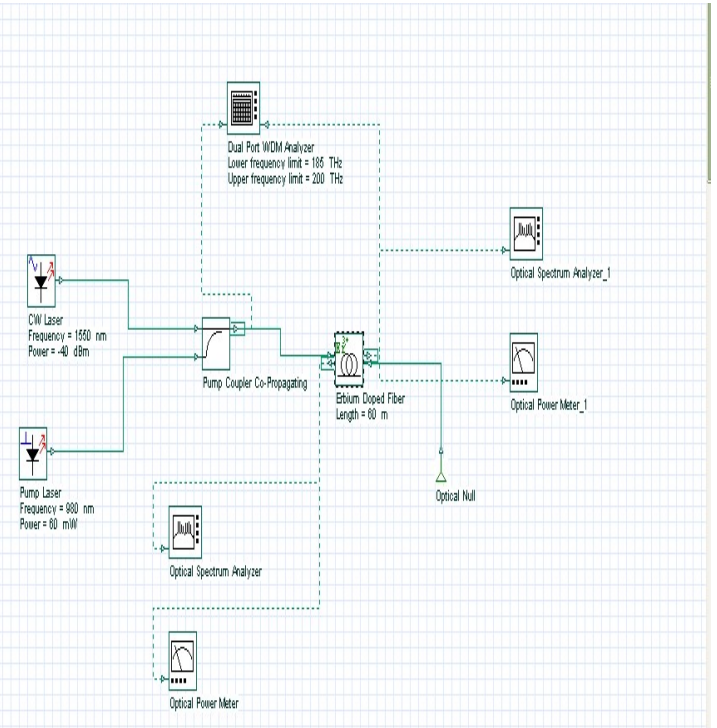

Fig 4 EDFA simulation setup

\begin{tabular}{|c|c|c|c|c|}
\hline Disp & Ilame & Value & Units & Mode \\
\hline$\sqrt{\nabla}$ & Length & 60 & $m$ & Normal \\
\hline$\Gamma$ & Er metastable lifetime & 10 & $m s$ & Normal \\
\hline$\Gamma$ & Input data & Fiber specification & & Normal \\
\hline Г & Saturation parameter & $4.4 e+015$ & $1 /(s . m)$ & Norma! \\
\hline Г & Core radius & 1.2 & $u m$ & Normal \\
\hline Г & Er doping radius & 1.2 & $u m$ & Normal \\
\hline Г & Er ion density & $1 e+025$ & $m^{n}-3$ & Normal \\
\hline$\Gamma$ & Ilumerical aperture & 0.32 & & Normal \\
\hline
\end{tabular}

Fig. 5 EDFA Properties

\subsection{Gain Characteristics}

The change of gain with fiber length has been displayed in figure (6.a) for several pump powers. There is variation in gain with the fiber length due to pump power changes. When the length is same, initially there is an exponential rise with the pump power and thereafter a decrease is observed beyond a specific amount. If pump power is fixed, there is an increase in amplifier gain till a specific fiber length. It then reaches a maximum after which it decreases. The gain decreases because the population inversion is inadequate and the losses are high.

\section{VARIATION OF GAIN WITH FIBRE LENGTH}

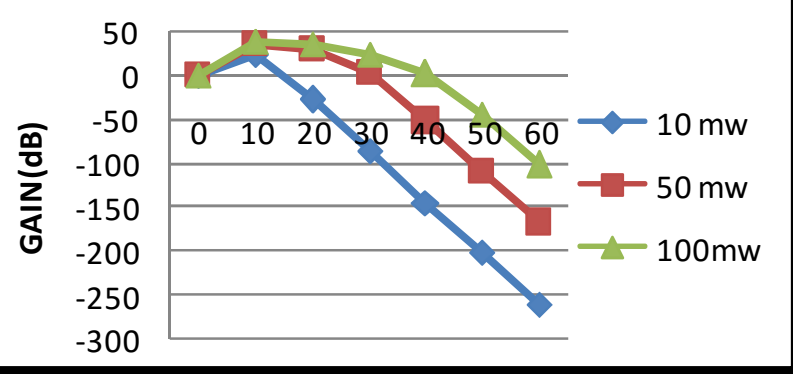

Fig 6(a) Variation of gain(dB) with Fiber Length(m) 


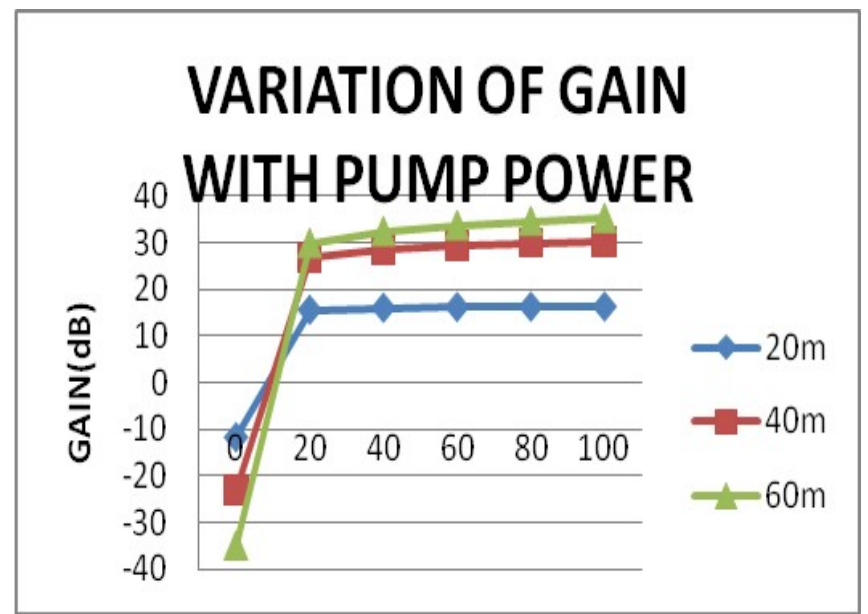

Fig 6(b) Variation of gain(dB) with Pump Power(mW)

Figure above displays the change of gain with pump power for several lengths $(20,40,60 \mathrm{~m})$. The pump power is changed from (0 to 100) mw and Er3+ density is (100ppmwt).

The gain increases with the increase in pump power. But beyond a specific amount of gain, there is smaller rise in gain and ultimately it leads to saturation. Also, it can be seen that, gain is higher for longer length of fiber.

\subsection{NOISE CHARACTERISTICS}

The change of noise figure with fiber length has been displayed in figure 7(a) for separate pump powers when input power and Er3+ density( 1000ppm-wt) are kept fixed. The noise figure rapidly increases with length. This is because the gain reduces as pump reduces suddenly.

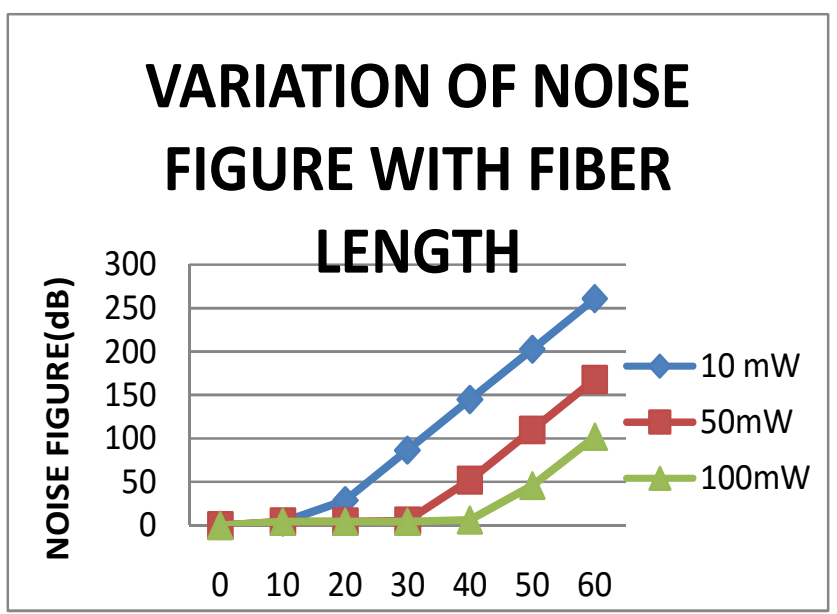

Fig 7(a) Change of $\mathrm{NF}(\mathrm{dB})$ with Fiber Length(m)

Figure (7.b) displays the noise figure changes with pump power for several fiber lengths $(20,40$ and60m) . It is observed that the noise figure is decreasing with an increase in pump power. When the pump power is less, the noise figure is greater for a long fiber than it is for a short fiber. It happens because pump power is inadequate to achieve large gain in an active fiber. When gain is large and the state is such that there are more atoms in excited state than in lower state, then the transition from excited state to lower state with emission of energy that is not in phase with incident energy does not increase. There is a linear relation between the noise figure of EDFA and Amplified Spontaneous Emission (ASE) power and inverse relation between noise and the amplifier gain; thus, if gain is increased then the NF of EDFA could be brought to a low level.

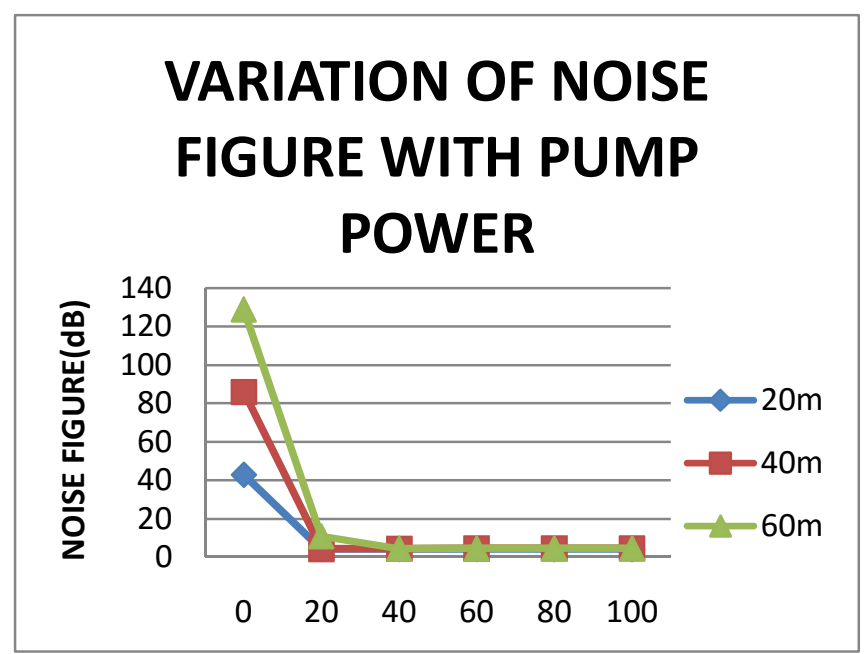

Fig 7 b Change of NF (dB) with pump power(mW)

\section{CONCLUSIONS}

In this paper, we introduced EDFA ,studied its principle and studied the characteristics of EDFA operation in Conventional band whose pumping is done at $980 \mathrm{~nm}$. Variations of Gain and noise figure were acquired in terms of fiber length and pump power.

The gain changes with the fiber length because there is variation in pump power. If the EDFA is provided with adequate pump power, it is seen that EDFA is employed in saturated regions which leads to large gain and low noise figure.

\section{REFERENCES}

[1]. Banaz O.Rashid, Perykhan.M.Jaff "Gain and Noise Figure Performance of Erbium Doped Fibre Amplifiers at $10 \mathrm{gbps}$ "

[2]. C Randy Giles and Emmaneul Desurvie,"Modelling Erbium Doped Fibre Amplifiers".IEEE Journal of Lightwave Technology,Volume 9 Issue2; Feb 1991 ; Pgs 271-283

[3]. Mishal singla,Preeti,Sanjiv "Comparative Analysis of EDFA based 64 channel WDM systems for different pumping techniques" International Journal of Scientific \& Engineering Research, Volume 5, Issue 6, June-2014

[4]. Sunil Kumar Panjeta,Onkar Chand,Dhanvir Mandal;"Gain Optimization of EDF Optical Amplifier by stages Enhancement and Variation In Input Pumping Power" International Journal Of Scientific and Research Publications, Volume 2,Issue11, Nov 2012 
[5]. Usman J Sindhi1, Rohit B Patel, Kinjal A Mehta1 and Vivekananda Mishra;'Performance Analysis of 32- channel WDM system using Erbium Doped Fibre Amplifier" International Journal Of Electrical and Electronic Engineering and Telecommunications; vol 2 April 2013

[6]. Murat Ari , Haldun Goktas,M Cengiz TAplamacioglu,: “Analysis Of Erbium Doped Fibre Amplifiers" 